\title{
A Brief Update on The Physiopathology of Atopic Dermatitis
}

\author{
Cláudia Silveira Baena, Julie Guimarães Hoyer and Zamir Calamita* \\ Marília Medical School, Monte Carmelo Street, Brazil \\ Submission: March 11, 2019; Published: March 25, 2019 \\ *Corresponding author: Zamir Calamita, Silvio Marinho Street, 405- Jardim Tangará, Marília - SP, Brazil
}

\begin{abstract}
Atopic dermatitis is a multifactorial allergic disease with a genetic component. Its main symptoms are intense pruritus, erythema, crust formation and exudation, as well as comorbidities such as insomnia, stress, mood changes and impaired concentration. Alterations in the skin barrier as well as disorders of the innate and adaptive immune response are the central components of the pathophysiology of the disease. The clinical condition can become a burden for the patient, making it essential to fully understand the pathophysiology of the disease. In this regard, the complete understanding of the physiopathology of the disease becomes essential for the diagnosis and for a complete biopsychosocial approach of the patient. This review, therefore, seeks to elucidate the latest AD updates regarding its pathophysiology.
\end{abstract}

Keywords: Atopic Dermatitis; Physiopathology

\section{Introduction}

Atopic dermatitis (AD) is a chronic inflammatory skin disease, with a hereditary, multifactorial and allergic character that develops in outbreaks $[1,2]$. Approximately $70 \%$ of patients with $\mathrm{AD}$ have a family history of atopy - such as asthma and allergic rhinosinusitis [3]. Their prevalence varies between countries, and specific factors in each region, such as poverty, genetics, helminth parasite infestation, and diverse flora may play a role in the development of this and other allergic diseases [4]. Generally, the disease begins before the age of 2 years, and may also occur at any time in life. In most patients, symptoms disappear before puberty, sometimes extending into adulthood [5]. The main symptoms of $\mathrm{AD}$ include erythema, edema, excoriations, crust formation, and exudation [1]. This clinical picture may lead to comorbidities, such as insomnia, stress, mood alteration, impairment in concentration and social stigmatization, transforming the pathology into a burden for the patient and family [1,6]. In this sense, a complete understanding of the risk factors as well as the pathophysiology of the disease becomes essential for the diagnosis and for a complete biopsychosocial approach of the patient.

\section{Physiopathology}

Skin Barrier Changes: The body's first defense against external agents is the skin barrier, consisting of cellular mechanical barrier, lipid mantle, acid mantle and resident flora. The mechanical cutaneous barrier is located in the stratum corneum - formed by corneocytes and lipids - and is responsible for avoiding penetration of allergens and excessive loss of transepidermal water. The corneocyte proteins, such filaggrin, and its main lipid component - ceramide - are responsible for water retention in the intercellular space [7]. Filagrin has metabolites components of the Natural Hydration Factor (FNH), necessary for the hydration and maintenance of the $\mathrm{pH}$ of the stratum corneum [7]. The acidic $\mathrm{pH}$ of the skin keeps the resident bacterial flora normal, making it difficult to colonize pathogenic bacteria mainly S. aureus [7]. The genetic alterations of AD lead to defects in the metabolism of filaggrin, compromising the $\mathrm{FNH}$ and the maintenance of the normal $\mathrm{pH}$ of the skin $[7,8]$. In addition, there is a reduction in the ceramide, leading to a loss of transmembrane water [7]. Other genetic alterations lead to lower expression of CLAUDIN1 - adhesion protein located in the occlusion junctions $[7,8]$. These changes predispose to infections and inflammation due to impaired skin barrier function [6].

Immune System Changes: Once the cutaneous barrier is ruptured, the innate immune response is initiated [7]. In AD, there is a decrease in TLR in keratinocytes and APCs. These receptors release antimicrobial peptides, chemokines and cytokines when stimulated, reinforcing the intercellular junctions and limiting infections $[7,8]$. This decrease, therefore, facilitates skin infections [9]. Regarding adaptive immunity, the pathogenesis of AD is permeated by a T cell mediated imbalance. Physiologically, dendritic cells and Langerhans cells recognize antigens and promote the induction of immune response of Th1, Th2, Th17, and Th22 lymphocytes. In the acute phase of $\mathrm{AD}$, prolonged exposure to pathogenic antigens, as well as thymic stromal lymphopoietin (TSLP) and innate lymphoid type 2 (ILC2) cells - increased in AD favor the Th2 lymphocyte lineage, which produce interleukins IL- 
4, IL-13 and IL-31, characteristics of the disease. The production and interaction of cytokines produced by Th2, Th22 and Th17, such as IL-4, IL-13, IL-22 and IL-17, end up inhibiting the epidermal differentiation of gene products, such as Filagrin [7].

Among the interleukins produced, IL-31 plays a key role in the induction of severe pruritus, whereas IL-22 contributes to epidermal hyperplasia. In the chronic phase of $\mathrm{AD}$, response of Th1 cells predominates, which produces IFN- $^{\gamma}$. This cytokine activates phagocytic cells and is related to skin barrier function, by inducing the modified differentiation of keratinocyte, characterizing tegument hypertrophy in AD [7]. Recent studies have also shown that ILC2s appear to express the natural killer cell-like lectin like receptor (KLRC1), which binds to E-cadherin - an epithelial adhesion molecule in AD that directly inhibits ILC2 proliferation and the production of IL-5 and IL-13. This suggests that healthy skin inhibits the function of ILC2, and the decrease in its interaction with E-cadherin leads to the inflammatory lack of control seen in $\mathrm{AD}$ [9].

\section{Conclusion}

$\mathrm{AD}$ is a common chronic dermatological condition that arises due to failure in the skin barrier, eosinophilic infiltration and high levels of IgE, resulting from a complex interaction between environmental factors, metabolic factors, genetic susceptibility, alterations of the skin barrier and immune system, as well as infectious processes. It is a pathology that causes great burden to the patient, mainly due to pruritus and visible excoriations. In this sense, studies that seek to elucidate the correct pathophysiology of D.A. are important, in order to bring benefits to patients and their families.

\section{References}

1. Hajar, Tamar, Gontijo João Renato Viannahanifin, Jon M (2018) New and Developing Therapies for Atopic Dermatitis. Brazilian Annals of Dermatology 93(1): 104-107.
2. Sánchez Jorge, Toro Yuliana, cardona Ricardo (2017) Clinical Impact in the Real Life of Guidelines Recommendations for Atopic Dermatitis in a Tropical Population (TECCEMA cohort). Allergy Mexico Journal 64 (3): 260 .

3. Mesquita Kleyton De Carvalho, Igreja Ana Carolina De Souza Machadocosta, Izelda Maria Carvalho (2013) Atopic Dermatitis and Vitamin D: Facts and Controversies. Brazilian Annals of Dermatology 88(6): 945-953.

4. Bruscky Dayanne Mota Veloso, Melo Ana Carolina Cavalcanti Dela Bianca, Sarinho Emanuel Sávio Cavalcanti (2017) Cross-cultural Adaptation and Validation of the Itching Severity Scale in Children and Adolescents with Atopic Dermatitis. São Paulo Pediatrics Journal 35(3).

5. Sánchez Jorge, Sánchez Andréscardona, Ricardo (2017) Particular Characteristics of Atopic Eczema in Tropical Environments. The Tropical Environment Control for Chronic Eczema and Molecular Assessment (TECCEMA) cohort study. Brazilian Annals of Dermatology 92(2): 177-183.

6. Campos Amanda Letícia Bezerra, Araújo Filipe Moreira, Desantos, Maria Amélia Lopes Dos (2017) Impact if Atopic Dermatitis on the Quality of life of Pediatric Patients and their Guardians. São Paulo Pediatrics Journal 35(1): 5-10.

7. Antunes, Adriana, Sole, Dirceu, Carvalho,Cania O, Bau, et al. (2017) Updated Practical Guide on Atopic Dermatitis - Part I: Etiopathogenesis, Clinical Features, and Diagnosis. Joint Position Paper of the Brazilian Association of Allergy and Immunology and the Brazilian Society of Pediatrics. Archives of Asthma, Allergy and Immunology.

8. Orfali, Raquel Leão (2015) Evaluation of the Effect of Staphylococcal Enterotoxins A and B in Th17, Th22 and CD38+ Cells in Adult Atopic Dermatitis. Doctoral Thesis, São Paulo University (USP).

9. Dohert Ta, Broide Dh (2015) Group 2 Innate Lymphoid Cells: New Players in Human Allergic Diseases. Journal investing Allergology Clinical Immunology 1(25): $1-11$. 